\title{
KINESIO TAPING NÃO ALTERA A RELAÇÃO EMG ENTRE VASTO LATERAL E VASTO MEDIAL DURANTE MEIO-AGACHAMENTO
}

\author{
KINESIO TAPING DON'T CHANGE EMG RELATION BETWEEN VASTUS LATERALIS - \\ MEDIALIS IN HALF SQUAT
}

KINESIO TAPING NO ALTERA LA RELACIÓN EMG ENTRE VASTO LATERAL Y VASTO MEDIAL DURANTE MEDIO AGACHAMIENTO

\begin{abstract}
Julio Cerca Serrão (Educador Físico)

João Gustavo Claudino'

(Educador Físico)

Pedro Luis Sampaio Miyashiro'

(Fisioterapeuta)

Bruno Mezêncio

(Educador Físico)

Rafael Soncin ${ }^{1}$

(Educador Físico)

Eric Pomi ${ }^{1}$

(Educador Físico)

Eduardo Borges ${ }^{1}$

(Educador Físico)

Miranne Cardoso da Silva ${ }^{1,2}$

(Educador Físico)

Igor Philip Gloria ${ }^{3}$ (Fisioterapeuta)

Vinicius Zanetti ${ }^{3}$ (Educador Físico)

Luis Mochizuki' (Educador Físico)

Alberto Carlos Amadio'

(Educador Físico)

1. Universidade de São Paulo,

Escola de Educação Física

e Esporte, Laboratório de

Biomecânica, São Paulo, SP, Brasil.

2. Minas Tênis Clube,

Departamento de Academia,

Setor de Ginástica Coletiva, Belo

Horizonte, MG, Brasil.

3. Red Bull Brasil, Departamento de

Saúde e Performance, Campinas,

SP, Brasil.
\end{abstract}

\section{Correspondência:}

Laboratório de Biomecânica. Escola de Educação Física e Esporte Universidade de São Paulo Av. Professor Mello Moraes, 65, Cidade Universitária, São Paulo, SP, Brasil. 05508-030.

jcserrao@usp.br

\section{RESUMO}

Introdução: Kinesio Taping é uma técnica realizada com aplicação de fitas elásticas sobre a pele, que se propõe a produzir determinados efeitos com fins de prevenção e tratamento das lesões musculoesqueléticas. No entanto, os meios pelos quais tais efeitos ocorrem continuam sendo investigados e discutidos, principalmente no que diz respeito à utilização no campo da reabilitação e do esporte. Objetivo: Analisar a relação da atividade eletromiográfica encontrada nos músculos vasto lateral (VL) e vasto medial (VM) em duas condições: sem aplicação de Kinesio Taping (GnKT) e com aplicação de Kinesio Taping (GKT) em uma população saudável, com experiência em treinamento de força. Métodos: Dezoito sujeitos do sexo masculino (idade: $28,1 \pm 6,9$ anos; massa corporal: $85,5 \pm 8,3 \mathrm{~kg}$; estatura: $179,5 \pm 6,9 \mathrm{~cm}$; comprimento de membro inferior: $97,0 \pm 4,2 \mathrm{~cm}$ ) realizaram o exercício de meio-agachamento livre, com velocidade controlada, sem e com aplicação de Kinesio Taping. A relação foi verificada pela proporção de magnitude de ativação (VMNL), utilizando-se os valores de root mean square (RMS). A sequência para realização dos exercícios nas condições mencionadas foi randomizada e balanceada. Resultados: Os valores encontrados para a razão VMNL na situação GnKT foram de 83,96 $\pm 5,79 \%$ para VM e 84,13 $\pm 7,16 \%$ para VL. Já na situação GKT, 84,55 \pm 16,97\% para VM e 80,53 \pm 9,20\% para VL. Não foram observadas diferenças significativas nos valores de RMS para a relação VMNL submetidos a aplicação de Kinesio Taping. Conclusão: A aplicação de Kinesio Taping não demonstrou influenciar a relação da atividade eletromiográfica entre os músculos vasto lateral e vasto medial durante a execução do exercício de meio-agachamento.

Descritores: exercício; eletromiografia; fita atlética.

\section{ABSTRACT}

Introduction: Kinesio Taping is a technique carried out with application of elastic tapes on the skin, which is intended to produce certain effects on prevention and treatment of musculoskeletal injuries. However, the means by which these effects occur are still being investigated and discussed, particularly with regard to rehabilitation and sport. Objective: To analyze the relationship of the electromyographic activity found in the vastus lateralis (VL) and vastus medialis (VM) muscles on two conditions: without the application of Kinesio Taping (GnKT) and with the application of Kinesio Taping (GKT) in a healthy population with experience in strength training. Methods: Eighteen male subjects (age: $28.1 \pm 6.9$ years, body mass: $85.5 \pm$ $8.3 \mathrm{~kg}$, height: $179.5 \pm 6.9 \mathrm{~cm}$, length of lower limb: $97.0 \pm 4.2 \mathrm{~cm}$ ) performed the exercise of free half-squat with controlled speed with and without applying Kinesio Taping. The relationship was verified by the ratio of activation magnitude (VMNL) using the root mean square (RMS). The sequence for the exercises under such conditions was randomized and balanced. Results: The values for the ratio VMNL in the GnKT situation were $83.96 \pm 5.79 \%$ for VM and $84.13 \pm 7.16 \%$ for VL. In the GKT situation, $84.55 \pm 16.97 \%$ for VM and $80.53 \pm 9.20 \%$ for VL. No significant differences were observed in RMS values for the VMNL ratio when the Kinesio Taping was applied. Conclusion: The application of Kinesio Taping did not affect the electromyographic ratio between the vastus lateralis and vastus medialis muscles during the half-squat exercise.

Keywords: exercise; electromyography; athletic tape.

\section{RESUMEN}

Introducción: Kinesio Taping es una técnica realizada con aplicación de cintas elásticas sobre la piel, que se propone producir determinados efectos con fines de prevención y tratamiento de las lesiones musculoesqueléticas. Sin embargo, los medios por los que tales efectos ocurren continúan siendo investigados e discutidos, principalmente en lo que refiere al uso en el campo de la rehabilitación y del deporte. Objetivo: Analizar la relación de la actividad electromiográfica encontrada en los músculos vasto lateral (VL) y vasto medial (VM) en dos condiciones: sin aplicación de Kinesio Taping (GnKT) y con aplicación de Kinesio Taping (GKT) en una población saludable, con experiencia en entrenamiento de fuerza. Métodos: Dieciocho sujetos del sexo masculino (edad:28,1 $\pm 6,9$ años; masa corporal: 85,5 $\pm 8,3 \mathrm{~kg}$; estatura: 179,5 $\pm 6,9 \mathrm{~cm}$; longitud de miembro inferior: $97,0 \pm 4,2 \mathrm{~cm}$ ) realizaron el ejercicio de medio agachamiento libre, con velocidad controlada, sin y con aplicación de Kinesio Taping. La relación fue verificada por la proporción de magnitud de activación (VMNL), utilizándose 
los valores de root mean square (RMS). La secuencia para realización de los ejercicios en las condiciones mencionadas fue aleatorizada y balanceada. Resultados: Los valores encontrados para la razón VMNL en la situación GnKT fueron de $83,96 \pm 5,79 \%$ para VM y $84,13 \pm 7,16 \%$ para VL. Ya en la situación GKT, 84,55 $\pm 16,97 \%$ para VMy 80,53 $\pm 9,20 \%$ para VL. No fueron observadas diferencias significativas en los valores de RMS para la relación VMNL sometidos a la aplicación de Kinesio Taping. Conclusión: La aplicación de Kinesio Taping no demostró influenciar la relación de la actividad electromiográfica entre los músculos vasto lateral y vasto medial durante la ejecución del ejercicio de medio agachamiento.

\section{Descriptores: ejercicio; electromiografía; cinta atlética.}

\section{INTRODUÇÃO}

O kinesio taping (KT) consiste em uma técnica que se utiliza da aplicação de uma fita elástica para o tratamento de lesões musculoesqueléticas oriundas da prática esportiva e de outras condições variadas ${ }^{1}$. Desenvolvido pelo quiropraxista japonês Dr. Kenso Kase em 1970, o KT tornou-se popular após ser largamente utilizado por atletas nos Jogos Olímpicos em $2008^{2}$. Dentre os efeitos diretos já mencionados pela literatura encontram-se a facilitação proprioceptiva ${ }^{3,4}$ a facilitação muscular ${ }^{5}$, a redução da fadiga muscular ${ }^{6}$, a redução de dor muscular tardia ${ }^{7}$ e a inibição da dor ${ }^{8,9}$.

Adicionalmente, de acordo com a sua referência teórica, Kase et al. ${ }^{10}$, sugere a efetividade da aplicação do KT para aumento da atividade eletromiográfica (EMG). Deste modo, sua aplicação poderia potencializar a EMG em articulações que demandam um fino equilíbrio muscular para otimizar o rendimento e/ou reduzir o risco de lesão. Favorecendo assim, articulações como o joelho, que possuem um dos mais elevados índices de lesão no aparelho locomotor. Os tipos de lesão no joelho encontrados clinicamente podem ser generalizados dentro das seguintes características: lesões meniscais, ligamentares, cartilaginosas, distensões inespecíficas e outras lesões menores, incluindo as por esforços de repetição ${ }^{11}$. Um dos fatores protetores para essas lesões é a estabilização da patela, assegurada pelos estabilizadores transversos, com a tração do vasto lateral (VL) e vasto medial (VM) ${ }^{12}$ e pelos estabilizadores longitudinais, formados pelos tendões do quadríceps e da patela. No entanto, alterações na geometria de aplicação de força no tendão podem ocasionar a lateralização desta estrutura anatômica ${ }^{13}$. O desequilíbrio na força ${ }^{14} \mathrm{Ou}$ ativação EMG de VL e VM ${ }^{15-21}$, e até mesmo uma menor área de secção transversa do VM²2, são apontados como possíveis causas da síndrome da dor patelofemoral (SDPF), lesão que atinge um número expressivo de indivíduos praticantes regulares de atividade física e atletas ${ }^{15,20,23}$.

Diante de tais fatos, intervenções com o KT têm sido bastante observadas no meio da atividade esportiva competitiva e de reabilitação. Apesar do uso frequente e disseminado no esporte e em atividade física, e da constante exposição dos supostos efeitos benéficos provenientes da técnica, há muito que ser investigado, uma vez que, as consequências mencionadas são muitas, mas os mecanismos que as explicam não são claramente eliciados. Além disso, não há evidências capazes de suportar tais repercussões².

Parreira et al. ${ }^{24}$ realizaram uma revisão sistemática, na qual analisaram estudos que se propuseram a verificar a eficiência do uso do KT em condições musculoesqueléticas diversas, tais como afecções da coluna lombar, joelho e ombro. Em nenhum dos trabalhos analisados há consistente evidência de que a técnica demonstra resultados satisfatórios, não justificando o seu uso em âmbito clínico. Williams et al. ${ }^{2}$ também dedicaram-se a estudar os possíveis efeitos da bandagem KT a partir de uma meta-análise, e corroboram com os achados propostos por Parreira et al. ${ }^{24}$ demonstrando a ausência de evidências para condições como dor musculoesquelética, propriocepção e nível de atividade muscular. A mesma conclusão chegaram Lim et al. ${ }^{25} \mathrm{em}$ sua meta-análise, demonstrando que o KT não apresentou ser uma solução para distúrbios osteo-mio-articulares e para a dor. Tal conclusão é ainda reforçada por Mostafavifar et al. ${ }^{26}$ em sua revisão sistemática.

Não foram encontrados estudos que verificaram a efetividade da aplicação do KT sobre a relação da atividade muscular entre os músculos VM e VL, tão pouco, como forma de aumentar a estabilização da articulação patelofemoral. Sendo assim, o presente estudo tem como objetivo analisar a relação entre VL e VM submetidos aplicação do KT em uma população saudável, livre de alterações do mecanismo extensor do joelho.

\section{MATERIAIS E MÉTODOS}

A amostra constituiu-se de 18 sujeitos do sexo masculino (idade: 28,1 $\pm 6,9$ anos; massa corporal: 85,5 $\pm 8,3$ kg; estatura: 179,5 56,9 cm; comprimento de membro inferior: $97,0 \pm 4,2 \mathrm{~cm}$ ) com pelo menos um ano experiência em treinamento de força, e que executam o exercício de meio agachamento livre por pelo menos uma vez por semana a no mínimo seis meses. Todos os sujeitos possuíam idade entre 20 e 40 anos e não possuíam distúrbios musculoesqueléticos nos últimos seis meses. O projeto foi aprovado pelo Comitê de Ética da unidade sob o protocolo Caae 37502214400005391 e todos os participantes assinaram o Termo de Consentimento Livre e Esclarecido.

Foi analisada a relação da root mean square (RMS) entre os músculos VM e VL durante a execução de 2 séries de 8 repetições do exercício meio agachamento livre, com velocidade controlada, sendo 1 segundo para cada fase do movimento. A relação foi expressa como a proporção de magnitude de ativação (VMNL). Onde valores superiores a 1,0 indicam uma maior atividade do VM em relação ao VL durante a execução do exercício. Também foi analisada a mediana da frequência (MF) dos músculos VM e VL nas diferentes situações, para verificar uma possível influência de fadiga.

A primeira foi realizada série sem aplicação do kinesio taping ${ }^{\circledR}$ (GnKT) e a segunda série com aplicação (GKT). Esta ordem foi adotada para que os valores obtidos em GnKT pudessem ser utilizados para normalização dos dados de EMG. A aplicação de KT quando necessária foi de acordo com o proposto por Kase et al. ${ }^{10}$, com a colocação da fita sobre o músculo quadríceps para aumento da ativação EMG, utilizando o tipo de aplicação facilitação (i.e., tensão de $20 \%$ a partir da extremidade proximal).

O exercício foi realizado com amplitude de $90^{\circ}$ de flexão de joelho, distância entre os pés, equivalente à largura biacromial e carga equivalente a 8 repetições máximas (RM) informada pelo próprio voluntário. A carga da execução das 8 RM para esta amostra era de 108,6 $\pm 30,9$ kg. A cadência do exercício foi controlada em um segundo para cada fase do movimento: excêntrica, concêntrica e tempo entre as repetições, e o intervalo entre as séries foi de 5 minutos.

Para aquisição do sinal EMG foi utilizado o sistema Noraxon Telemyo 2400 GT2 (Arizona, USA), com taxa de amostragem de $3000 \mathrm{~Hz}$, conversor analógico-digital de 16 bits. Os eletrodos foram posicionados nos músculos VM e VL de acordo com recomendação da SENIAM. Para normalização do sinal EMG foi utilizado o maior valor RMS obtido dentre as fases de cada repetição da primeira série do exercício. O sinal eletromiográfico foi filtrado com filtro passa-banda do tipo Butterworth de quarta ordem com frequência de corte de 10 e $450 \mathrm{~Hz}$. 
A normalidade dos dados foi verificada pelo teste de Komolgorov-Smirnov. Para comparação dos parâmetros EMG foi utilizado o Teste $t$ pareado. Adicionalmente, a relação entre VM e VL foi analisada utilizando uma abordagem contemporânea denominada de inferência baseada em magnitude ${ }^{27}$ a fim de detectar pequenos efeitos de importância prática para o ambiente aplicado. Foi utilizada uma planilha proposta pelo autor para estabelecer a probabilidade de cada manipulação experimental tendo um efeito classificado como benéfico, negligenciável ou danoso. Descritores qualitativos foram atribuídos aos escores percentuais quantitativos da seguinte forma: $<1 \%$ quase certamente não; $1-5 \%$ muito improvável; $5-25 \%$ improvável; $25-75 \%$ possível; 75-95\% provável; 95-99\%, muito provável,>99\% quase certamente sim. Se mudanças benéficas e danosas fossem simultaneamente $>5 \%$, a inferência era considerada inconclusiva ${ }^{28,29}$. O nível de significância adotado foi de $p<0,05$ e as análises foram realizadas no software SigmaStat 3.5.

\section{RESULTADOS}

Para a situação GnKT foi encontrado valores de RMS de 83,96 \pm $5,79 \%$ para VM e 84,13 \pm 7,16\% para VL, já na situação GKT, 84,55 \pm $16,97 \%$ para VM e 80,53 $\pm 9,20 \%$ para VL. Não foram encontradas diferenças significativas entre as situações GnKT e GKT para atividade EMG dos músculos VM $(p=0,900)$ e VL $(p=0,122)$. A relação entre os músculos VL e VM não apresentou diferença significativa $(p=0,292)$ para as situações GnKT e GKT (Figura 1). Adicionalmente, não foi verificada nenhuma alteração significativa nos valores de MF para o VM (GnKT: $62,17 \pm 11,94 \mathrm{~Hz} ; \mathrm{GKT}: 62,96 \pm 11,50 \mathrm{~Hz} ; \mathrm{p}=0,744$ ) e para o VL (GnKT: 60,17 $\pm 8,29 \mathrm{~Hz} ; \mathrm{GKT}: 62,42 \pm 10,89 \mathrm{~Hz} ; \mathrm{p}=0,542$ ).

A análise de inferência baseada na magnitude suporta os resultados encontrados de que não houve efeito da aplicação do KT nas variáveis EMG analisadas. Para a atividade EMG do músculo VM a utilização do KT resultou em um efeito muito provavelmente negligenciável (95,3\%) no RMS, com uma probabilidade de 2,9\% de que ocorra um aumento do RMS e uma probabilidade de 1,8\% de que ocorra uma redução do RMS. Já no músculo VL houve um efeito provavelmente negligenciável $(88,3 \%)$, com uma probabilidade $<0,1 \%$ de que ocorra um aumento do RMS e uma probabilidade de $11,6 \%$ de que ocorra uma redução do RMS.

Na relação VM e VL foi encontrado um efeito provavelmente negligenciável $(88,6 \%)$ do KT para alterar este parâmetro. A probabilidade de que ocorra um aumento da relação é de 11,3\%, sendo encontrada uma probabilidade de 0,2\% de que ocorra uma redução da mesma.

Quanto a MF do músculo VM foi encontrado um efeito "quase certamente sim" negligenciável (99,8\%) para alteração desta variável, com $0,2 \%$ de probabilidade de aumento e não há probabilidade de redução. Sendo que para a MF do músculo VL foi encontrado um efeito provavelmente negligenciável (90,7\%), com 8,5\% de probabilidade de aumento e $0,8 \%$ de probabilidade de redução (Figura 2).

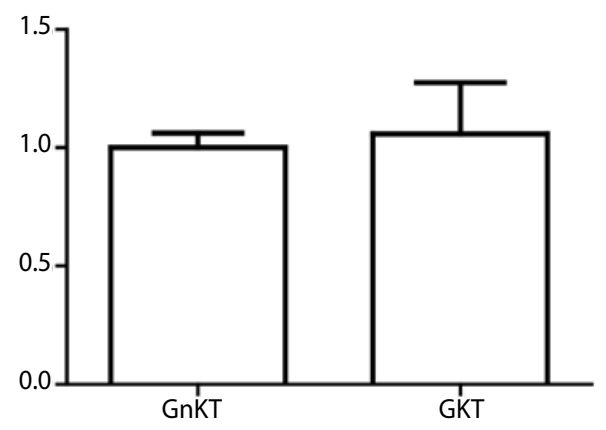

Figura 1. Relação entre os músculos: vasto medial e vasto lateral. GnKT = sem kinesio taping; GKT = com kinesio taping.

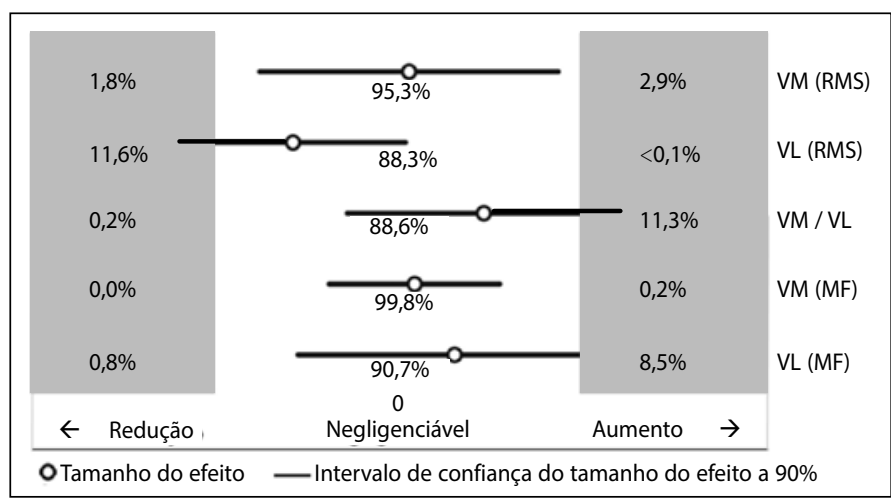

Figura 2. Resultado da análise de inferência baseada em magnitude. VM = vasto medial; RMS = root mean square; $\mathrm{VL}=$ vasto lateral; $\mathrm{MF}=$ mediana da frequência.

\section{DISCUSSÃO}

A investigação da atividade EMG tem sido utilizada para tentar buscar as possíveis modificações hipoteticamente induzidas pelo uso do KT. Até onde sabemos, este é o primeiro estudo que verificou a aplicação do KT como forma de alterar a relação VL e VM. Para esta investigação, foi hipotetizado que a modificação da técnica de aplicação do KT poderia alterar a relação VL e VM, e assim otimizaria a estabilização da articulação patelofemoral. No entanto, a aplicação proposta não alterou a relação entre VL e VM em homens treinados em musculação sem alterações ósteo-mio-articulares no joelho.

Uma das prováveis causas disto se daria pelo efeito do treinamento, uma vez que os sujeitos já apresentavam um destacado equilíbrio entre o VL (i.e., 49,9\%) e o VM (50,1\%). Tal equilíbrio não foi afetado pelas aplicações do KT (i.e., VL = 48,8\%; VM = 51,2\%). Esses níveis de atividade EMG equilibrados entre VL e VM são similares aos encontrados por Escamilla et al. ${ }^{30}$ que utilizaram o mesmo exercício de agachamento com carga de 12 RM, e encontraram ativação de aproximadamente 85\% da contração voluntária isométrica máxima (CVIM) para VM, e de 80\% da CVIM para VL em sujeitos saudáveis. A relação VMNL também foi investigada para exercícios diferentes. Irish et al..$^{31}$ avaliaram a relação VMNLL em cadeia cinética aberta e fechada, em sujeitos saudáveis, e as relações encontradas não indicaram predominância de atividade EMG para o VM ou o VL. Tais achados concordam com os resultados observados no presente estudo, onde em condições normais ocorre equilíbrio no recrutamento muscular, independente da aplicação de KT.

Nossos achados se associam a outros de revisões sistemáticas e meta-análises onde as evidências acerca dos benefícios do KT não foram encontradas ${ }^{2,24,25,26}$. No entanto, alguns estudos de caráter original apresentam resultados controversos. Pesquisadores utilizando a aplicação de KT sob o conceito de facilitação verificaram uma alteração da atividade EMG para o músculo gastrocnêmio medial durante a execução do salto vertical em comparação com o grupo placebo realizado com fita inextensível. Todavia, tal mudança não foi suficiente para alterar o desempenho do salto ${ }^{32}$. Isto descartaria um adicional efeito mecânico do KT. Adicionalmente, não foram encontradas diferenças significativas para a atividade EMG do músculo fibular longos de atletas de basquetebol submetidos à aplicação ativadora de KT, existindo aumento para o grupo placebo durante a realização do star excursion balance test ${ }^{33}$. Com achados semelhantes, Lins et al. ${ }^{34}$ demonstraram não haver alteração da atividade EMG para o quadríceps sob aplicação de KT, e nenhuma modificação no desempenho do hop test em indivíduos saudáveis sem histórico de lesões musculoesqueléticas. Tais evidências direcionam as investigações para descobrir outros mecanismos, não somente mecânicos, para explicar as modificações atribuídas ao KT, e até mesmo descritas por seus usuários, uma vez que, a atividade EMG não parece ser afetada sob as condições testadas.

A função do VM para estabilização da patela ocorre devido as suas 
fibras serem inseridas obliquamente na face superior e medial desta estrutura anatômica, formando um ângulo entre $45^{\circ}$ e 55\%, evitando assim, um excessivo deslocamento lateral da patela ${ }^{18,19}$. Como forma de apresentar uma das principais consequências práticas desse desequilíbrio muscular podemos citar a SDPF. Na literatura são encontrados estudos que relatam o desequilíbrio da atividade EMG dos músculos do quadríceps em voluntários com SDPF, sugerindo uma diminuição da magnitude ou atraso de ativação EMG do VM em relação ao VL, resultando no maior deslocamento lateral da patela 18,19,21,35,36. Diante disso, algum aparato que pudesse modificar a atividade EMG para mais ou para menos poderia ser empregado nesses casos. No entanto, essa implicação prática com o KT não foi efetiva para tal função baseando-se nos nossos achados e nos relatos da literatura já citados. Por outro lado, pesquisadores que não utilizaram o KT e também verificaram a relação da magnitude da atividade EMG de VM/VL em sujeitos saudáveis durante a subida e descida de escadas, comparando a com indivíduos acometidos pela SDPF ${ }^{37}$. Os resultados mostraram diferenças significativas entre as duas populações, com os sujeitos saudáveis apresentando maiores relações. Contrariamente, utilizando abordagens similares, não foram encontradas diferenças significativas na relação VMNL observada entre sujeitos saudáveis e com SDPF, ainda que sob a variação de exercícios e modalidades diferentes como em cadeia cinética aberta ou fechada ${ }^{38-40}$, apresentando nessa temática que resultados inconsistentes ainda existem na literatura.

Para verificar a validade do argumento de prevenção do KT, alguns trabalhos investigaram os efeitos decorrentes do seu uso em condições de não lesão. Nosaka observou menor tempo de recuperação e diminuição da dor muscular tardia em indivíduos saudáveis que receberam a aplicação do KT durante a realização de exercícios excêntricos para o membro superior, não indicando as razões para tais achados. Adicionalmente, Halseth et al. ${ }^{3}$ testaram a sensação proprioceptiva do tornozelo em uma amostra livre de lesões com e sem KT, resultando em nenhuma alteração entre as condições. Portanto, a utilização do KT com a justificativa de proporcionar a prevenção de lesões, ainda há dúvidas sobre como isto ocorre, tanto no que diz respeito à ativação muscular, quanto no que concerne a outras repercussões como a propriocepção articular e dor.

\section{CONCLUSÃO}

Os resultados demonstraram que o KT não alterou a relação da atividade EMG entre os músculos VL e VM durante a execução do exercício de meio agachamento. Além disso, a análise de inferência baseada em magnitude, verificou um efeito negligenciável da aplicação do KT quanto as "reais" alterações no desempenho.

\section{AGRADECIMENTO}

O presente trabalho foi realizado com o apoio da CAPES, entidade do governo brasileiro voltada para a formação de recursos humanos.

Todos os autores declararam não haver qualquer potencial conflito de interesses referente a este artigo.

CONTRIBUIÇÕES DOS AUTORES: Cada autor contribuiu individual e significativamente para o desenvolvimento do manuscrito. JCS (0000-0002-3646-3387)* participou substancialmente na concepção do trabalho. JGC (0000-0002-0263-8118)* e PLSM (0000-0002-5341-1327)* participaram no desenho do trabalho, aquisição, análise e interpretação dos dados e redação do artigo. BM (0000-0001-6293-5571)* e RS (0000-0001-5781-8928)* contribuíram no desenho, aquisição e análise estatística dos dados e interpretação dos achados. EP (0000-0003-4869-0568)* contribuiu na aquisição gerenciamento das coletas e instrução aos voluntários. EB (0000-0002-7815$1365)^{*}$ contribuiu na aquisição e análise dos dados e redação. MCS (0000-0002-1356-2446)* IPG (0000-0002-4486-8616)* e VZ (0000-0001-9384-1658)* contribuíram na redação do artigo. LM (0000-0002-7550-2537)* contribuiu na análise estatística e interpretação dos dados. ACA (0000-0002-2527-2163)* participou da revisão crítica do conteúdo e aprovação final do manuscrito. Todos os autores contribuíram com o conceito intelectual do estudo.*Número ORCID (Open Researcher and Contributor ID).

\section{REFERÊNCIAS}

1. Thelen MD, Dauber JA, Stoneman PD. The clinical efficacy of kinesio tape for shoulder pain: a randomized, double-blinded, clinical trial. J Orthop Sports Phys Ther. 2008;38(7):389-95.

2. Williams $S$, Whatman $C$, Hume PA, Sheerin K. Kinesio taping in treatment and prevention of sports injuries: a meta-analysis of the evidence for its effectiveness. Sports Med. 2012;42(2):153-64.

3. Halseth T, McChesney JW, Debeliso M, Vaughn R, Lien J. The effects of kinesio ${ }^{T M}$ taping on proprioception at the ankle. J Sports Sci Med. 2004;3(1):1-7.

4. Jaraczewska $E$, Long C. Kinesio taping in stroke: improving functional use of the upper extremity in hemiplegia. Top Stroke Rehabil. 2006:13(3):31-42.

5. Hammer WI. Functional soft-tissue examination and treatment by manual methods. 3rd ed. Boston: Jones and Bartlett Publishers; 2006

6. Canina M, Ferrero V, Signaroll J. Wearability in the development of protection system for the lower limb. Disponivel em: http://www.phealth2008.org/Events/papers/p12.pdf. [Acessado em: 4/jul/2013]

7. Nosaka K. The effect of Kinesio taping on muscular micro-damage following eccentric exercises. In: Paper presented at the $15^{\text {th }}$ Annual Kinesio Taping International Symposium, Tokyo, 1999.

8. Kahanov L. Kinesio taping, Part 1: an overview of its use in athletes. Athl Ther Today. 2007;12(3):17-8.

9. Kneeshaw D. Shoulder taping in the clinical setting. J Bodyw Mov Ther. 2002;6:2-8.

10. Kase K, Wallis J, Kase T. Clinical therapeutic applications of the kinesio taping method. 2nd. Tokyo: Ken Ikai Co; 2003

11. Dutton M. Dutton's orthopaedic. examination, evaluation and intervention. 3rd ed. New York: McGraw Hill; 2012.

12. Bevilaqua-Grossi D, Felício LR, Simões R, Coqueiro KRR, Monteiro-Pedro V. Avaliação eletromiográfica dos músculos estabilizadores da patela durante o exercício isométrico de agachamento em indivíduos com síndrome da dor femoropatelar. Rev Bras Med Esporte. 2005;11(3):159-63.

13. Powers CM. The influence of altered lower-extremity kinematics on patellofemoral joint dysfunction: a theoretical perspective. J Orthop Sports Phys Ther. 2003;33(11):639-46.

14. Sakai N, Luo ZP, Rand JA, An KN. The influence of weakness in the vastus medialis oblique muscle on the patellofemoral joint: an in vitro biomechanical study. Clin Biomech (Bristol, Avon). 2000;15(5):335-9.

15. Petersen W, Ellermann A, Gösele-Koppenburg A, Best R, Rembitzki IV, Brüggemann GP, et al. Patellofemoral pain syndrome. Knee Surg Sports Traumatol Arthrosc. 2014;22(10):2264-74.

16. Pal S, Draper CE, Fredericson M, Gold GE, Delp SL, Beaupre GS, et al. Patellar maltracking correlates with vastus medialis activation delay in patellofemoral pain patients. Am J Sports Med. 2011;39(3):590-8.

17. Van Tiggelen D, Cowan S, Coorevits P, Duvigneaud N, Witvrouw E. Delayed vastus medialis obliquus to vastus lateralis onset timing contributes to the development of patellofemoral pain in previously healthy men: a prospective study. Am J Sports Med. 2009;37(6):1099-105.

18. Cowan SM, Bennell KL, Crossley KM, Hodges PW, McConnell J. Physical therapy alters recruitment of the vasti in patellofemoral pain syndrome. Med Sci Sports Exerc. 2002;34(12):1879-85.

19. Cowan SM, Bennell KL, Hodges PW, Crossley KM, McConnell J. Delayed onset of electromyographic activity of vastus medialis obliquus relative to vastus lateralis in subjects with patellofemoral pain syndrome. Arch Phys Med Rehabil. 2001;82(2):183-9.

20. Powers CM. Rehabilitation of patellofemoral joint disorders: a critical review. J Orthop Sports Phys Ther. 1998;28(5):345-54.

21. Witvrouw E, Sneyers C, Lysens R, Victor J, Bellemans J. Reflex response times of vastus medialis oblique and vastus lateralis in normal subjects and in subjects with patellofemoral pain syndrome. J Orthop Sports Phys Ther. 1996;24(3):160-5.

22. Pattyn E, Verdonk P, Steyaert A, Vanden Bossche L, Van den Broecke W, Thijs $Y$, et al. Vastus medialis obliquus atrophy: does it exist in patellofemoral pain syndrome? Am J Sports Med. 2011;39(7):1450-5

23. Blønd L, Hansen L. Patellofemoral pain syndrome in athletes: a 5.7-year retrospective follow-up study of 250 athletes. Acta Orthop Belg. 1998;64(4):393-400.

24. Parreira PCS, Costa LCMC, Junior LCH, Lopes AD, Costa LOP. Current evidence does not support the use of Kinesio Taping in clinical practice: a systematic review. J Physiother. 2014;60(1):31-9.

25. Lim EC, Tay MG. Kinesio taping in musculoskeletal pain and disability that lasts for more than 4 weeks: is it time to peel off the tape and throw it out with the sweat? A systematic review with meta-analysis focused on pain and also methods of tape application. Br J Sports Med. 2015;49(24):1558-66.

26. Mostafavifar M, Wertz J, Borchers J. A systematic review of the effectiveness of kinesio taping for musculoskeletal injury. Phys Sportsmed. 2012;40(4):33-40.

27. Hopkins WG. A spreadsheet for deriving a confidence interval, mechanistic inference and clinical inference from a p value. Sportscience. 2007;11:16-20.

28. Batterham AM, Hopkins WG. Making meaningful inferences about magnitudes. Int J Sports Physiol Perform. 2006;1 (1):50-7.

29. Hopkins WG. Probabilities of clinical or practical significance. Sportscience. 2002;6:1-2.

30. Escamilla RF, Fleisig GS, Zheng N, Barrentine SW, Wilk KE, Andrews JR. Biomechanics of the knee during closed kinetic chain and open kinetic chain exercises. Med Sci Sports Exerc. 1998;30(4):556-69.

31. Irish SE, Millward AJ, Wride J, Haas BM, Shum GL. The effect of closed-kinetic chain exercises and open-kinetic chain exercise on the muscle activity of vastus medialis oblique and vastus lateralis. Strength Cond Res. 2010;24(5):1256-62.

32. Huang CY, Hsieh TH, Lu SC, Su FC. Effect of the Kinesio tape to muscle activity and vertical jump performance in healthy inactive people. Biomed Eng Online. 2011;10:70

33. Briem K, Eythörsdöttir H, Magnúsdóttir RG, Pálmarsson R, Rúnarsdöttir T, Sveinsson T. Effects of kinesio tape compared with nonelastic sports tape and the untaped ankle during a sudden inversion perturbation in male athletes. J Orthop Sports Phys Ther. 2011:41(5):328-35.

34. Lins CA, Locks Neto F, Amorim AB, Macedo LB, Brasileiro JS. Kinesio Taping $\left({ }^{\oplus}\right)$ does not alter neuromuscular performance of femoral quadriceps or lower limb function in healthy subjects: randomized blind, controlled, clinical trial. Man Ther. 2013;18(1):41-5.

35. Chester R, Smith TO, Sweeting D, Dixon J, Wood S, Song F. The relative timing of VMO and VL in the aetiology of anterior knee pain: a systematic review and meta-analysis. BMC Musculoskelet Disord 2008:9:64

36. Crossley KM, Cowan SM, Bennell KL, McConnell J. Knee flexion during stair ambulation is altered in individuals with patellofemoral pain. J Orthop Res. 2004;22(2):267-74.

37. Souza DR, Gross MT. Comparison of vastus medialis obliquus: vastus lateralis muscle integrated electromyographic ratios between healthy subjects and patients with patellofemoral pain. Phys Ther. 1991;71(4):310-6.

38. Fehr GL, Cliquet Junior A, Cacho EWA, Miranda JB. Efetividade dos exercícios em cadeia cinética aberta e cadeia cinética fechada no tratamento da síndrome da dor patelofemoral. Rev Bras Med Esporte. 2006;12(2):66-70.

39. Laprade J, Culham E, Brouwer B. Comparison of five isometric exercises in the recruitment of the vastus medialis oblique in persons with and without patellofemoral pain syndrome. J Orthop Sports Phys Ther. 1998:27(3):197-204

40. McClinton S, Donatell G, Weir J, Heiderscheit B. Influence of step height on quadriceps onset timing and activation during stair ascent in individuals with patellofemoral pain syndrome. J Orthop Sports Phys Ther. 2007:37(5):239-44 